\title{
A method to estimate dietary total antioxidant capacity among elderly Irish women
}

\author{
C. Norton and P. Jakeman \\ Human Science Research Unit, Faculty of Education \& Health Sciences, University of Limerick, Limerick, \\ Republic of Ireland
}

Antioxidant intake is potentially protective against cell oxidative damage and related metabolic complications yet little is known of the dietary total antioxidant capacity (TAC) that might inform an RDA.

The aim of this study was to establish a WISPC(Tinuviel Software, Anglesey, UK) -based method of estimating dietary TAC founded on the USDA Database for the Oxygen Radical Absorbance Capacity (ORAC) of Selected Foods ${ }^{(1)}$ and to use this method to estimate the total dietary antioxidant capacity of a sample of elderly Irish women.

The descriptors of 326 foods listed in the USDA database were matched with foods in the WISPC database and their respective scores for total $(\mathrm{T})$, hydrophilic $(\mathrm{H})$ and lipophilic $(\mathrm{L})$-ORAC score (mmol Trolox equivalent/100 g; mmolTE), and the associated total phenolics score (mg Gallic acid equivalents/100 g; mgGAE) were assigned to 475 foods.

Using an estimated food intake record (eFIR) 75 healthy, elderly women provided 4-day food and drink consumption data that were analysed by WISPC (Tinuviel Software, Anglesey, UK). Following exclusion of invalid eFIRs, 53 records (mean (SD); age 61.1(5.3) y; BMI 24.9(4.6) kg/m²; BMR 1287(132) $\mathrm{kcal} / \mathrm{d})$ were analysed further.

Analysis of the nutrient intake showed mean TAC of 9.3(5.1) mmol TE (Table 1). TAC was further divided into its constituent hydrophilic $97(2) \%$ and lipophilic 3(2)\% components. The food groups contributing most to TAC were vegetables (23\%), fruit / fruit juices $(21 \%)$ and other beverages $(21 \%)$ with a lesser contribution from alcohol (12\%) and confectionary (7\%). By comparison, estimates of TAC, based upon USDA's Continuing Survey of Food Intake by Individuals (1994-1996) or more recently intake from food consumption data of NHANES 2001-02, was $5.6 \mathrm{mmol}$ TE and $4.7 \mathrm{mmol} \mathrm{TE}^{(2)}$.

Table 1

\begin{tabular}{|c|c|c|c|c|c|c|}
\hline & & Mean & Median & SD & 5th percentile & 95th percentile \\
\hline T-ORAC & $(\mathrm{mmol} \mathrm{TE})$ & 9.3 & 9.2 & 5.1 & 2.3 & 15.8 \\
\hline H-ORAC & (mmol TE) & 7.2 & 6.9 & 4.1 & 2.3 & 13.2 \\
\hline L-ORAC & (mmol TE) & 0.2 & 0.1 & 0.2 & 0 & 0.7 \\
\hline Total Phenolics & (mg GAE) & 2310 & 1331 & 2414 & 99 & 7067 \\
\hline$\% \mathrm{H}$ of $\mathrm{T}$-ORAC & $\%$ & $97 \%$ & $98 \%$ & $2 \%$ & $93 \%$ & $99 \%$ \\
\hline$\% \mathrm{~L}$ of T-ORAC & $\%$ & $3 \%$ & $2 \%$ & $2 \%$ & $1 \%$ & $7 \%$ \\
\hline
\end{tabular}

Oxidative stress is a key issue in many of the diseases of aging e.g. sarcopaenia, even if it is not a causative factor. There is continued growing interest in dietary TAC and in vivo antioxidant status and effects on health outcomes. Dietary TAC may be a potential marker of diet intake quality, providing a novel approach to assess the role of antioxidant intake on health, particularly among the elderly.

1. U.S. Department of Agriculture, Agricultural Research Service. 2010. Oxygen Radical Absorbance Capacity (ORAC) of Selected Foods, Release 2. Nutrient Data Laboratory Home Page: http://www.ars.usda.gov/nutrientdata/orac

2. Prior RL, Gu L, Wu X, Jacob RA, Sotoudeh G, Kader AA, Cook RA. (2007) Plasma antioxidant capacity changes following a meal as a measure of the ability of a food to alter in vivo antioxidant status. $J$ Am Coll Nutr 26(2), 170-81. 